\title{
CO LINE EMISSION IN PLANETARY AND PROTO-PLANETARY NEBULAE: THE MOLECULAR ENVELOPE
}

\author{
G. SILVESTRO AND I. PORRO
}

Istituto di Fisica Generale dell'Università, via P. Giuria 1, I-10125, Torino, Italy

Planetary nebulae (PNe) are believed to form when the envelope of gas and dust, ejected at the end of the AGB phase of stellar evolution, is heated by the hot stellar nucleus, left after mass loss due to the "superwind" is terminated. The presence of residual molecular gas in $\mathrm{PNe}$ should therefore be important for understanding the formation and subsequent evolution of the nebulae. Observations (Bachiller et al. 1988 ) of CO line radiation in proto-planetary nebulae (PPNe), the transition phase from AGB stars to PNe, allowed to set very low upper limits on the molecular gas mass and to predict almost complete dissociation of the molecular envelope at the end of the PPN stage. On the other hand, observations (Huggins \& Healy 1989; Healy \& Huggins 1990) of CO lines detected intense signals from several PNe, including some evolved sources.

The two apparently contradictory sets of data are critically discussed. We point out the uncertainties in the results for PPNe, due to the small number of surveyed sources and to the lack of well defined identification criteria for this class of objects. New methods are suggested, which could allow to increase the number of identified PPNe and thus obtain more reliable estimates for the molecular mass in their envelopes.

\section{References}

Bachiller, R., Gomez-Gonzales, J., Bujarrabal, V. \& Martin-Pintado, J. (1988) Astron. Astrophys. 196, L5

Healy, A.P. \& Huggins, P.J. (1990) Astron. J. 100, 511

Huggins, P.J. \& Healy, A.P. (1989) Astrophys. J. 346, 201 